\title{
CRANIOMETRIA EM LOBOS-GUARÁ Chrysocyon brachyurus ILLIGER, 1815 (CARNIVORA, CANIDAE)
}

\section{CRANIOMETRIC MEASUREMENTS IN MANED WOLVES Chrysocyon brachyurus ILLIGER, 1815 (CARNIVORA, CANIDAE)}

\author{
André Luiz Quagliatto Santos ${ }^{1^{*}}$ \\ Beatriz Furlan Paz ${ }^{1}$ \\ Rafael Ferraz Barros ${ }^{1}$ \\ Susana Faria Nalla ${ }^{1}$ \\ Thiago Souza Pereira² \\ 1Universidade Federal de Uberlândia, Uberlândia, MG, Brasil. \\ 2Instituto Estadual de Florestas, Uberlândia, MG, Brasil \\ *Autor para correspondência - quagliatto.andre@gmail.com
}

\section{Resumo}

Os objetivos do presente trabalho foram obter mensurações em crânios de Chrysocyon brachyurus adultos, caracterizar valores craniométricos para a espécie e observar se a classificação craniométrica de cães domésticos é adequada para o lobo-guará. Foram utilizados sete crânios de lobo-guará adultos, sem distinção de sexo. Determinou-se a localização de nove pontos craniométricos utilizados em cães domésticos para o lobo-guará. Os pontos foram referência para as 18 medidas craniométricas utilizadas para o cálculo de seis índices craniométricos. Os índices craniométricos são: cefálico 96,147 $\pm 3,89$ mm, crânio facial 1,06 $\pm 0,20 \mathrm{~mm}$, do neurocrânio $61,68 \pm 1,83 \mathrm{~mm}$, facial 100,06 $\pm 3,36 \mathrm{~mm}$, basal 29,79 \pm $1,18 \mathrm{~mm}$, e do forame magno $89,25 \pm 7,82 \mathrm{~mm}$. A classificação em braquicefálico, dolicocefálico ou mesaticefálico não se adequa a Chrysocyon brachyurus.

Palavras-chave: carnívoros; crânio; morfometria.

\begin{abstract}
The aim of this study was to obtain craniometric measures in adult Chrysocyon brachyurus skulls, establish craniometric values for the species, and determine if the craniometric classification of domestic dogs is suitable for the maned wolf. Seven skulls of adult maned wolves were used, with no gender distinction. Nine craniometric points used in domestic dogs were used for the maned wolf. The points served as reference to 18 craniometric measurements used subsequently to calculate six skull indices. Craniometric indeces were: cefalic $96.147 \pm 3.89 \mathrm{~mm}$, craniofacial $1.06 \pm 0.20 \mathrm{~mm}$, neurocranium $61.68 \mathrm{~mm} \pm 1.83$, facial $100.06 \pm 3.36 \mathrm{~mm}$, basal $29.79 \pm 1.18 \mathrm{~mm}$, and foramen magnum index of $89.25 \pm 7.82 \mathrm{~mm}$. The usual classification in brachicephalic, dolichocephalic, and mesaticephalic is not valid for Chrysocyon brachyurus.
\end{abstract}

Keywords: carnivores; morphometry; skull. 


\section{Introdução}

O crânio caracteriza-se por abrigar e proteger o encéfalo, bem como os orgãos dos sentidos especiais (visão, olfato, audição, equilíbrio e gustação), apresenta aberturas para passagens de ar e alimentos, além de maxilares e mandíbulas, incluindo os dentes para mastigação ${ }^{(1)}$.

O formato do crânio é um dos critérios mais importantes para determinar padrões raciais e modelos evolutivos $^{(2)}$. Diversos estudos já foram realizados em espécies domésticas como caprinos $^{(3)}$ e caninos $^{(2)}$. Christiansen e Adolfssen ${ }^{(4)}$ realizaram mensurações em 56 espécies de carnívoros, dentre felídeos, canídeos e ursídeos, cujo objetivo foi avaliar aspectos relacionados à força de mastigação. Em espécies silvestres, não foram encontrados estudos para determinar padrões craniométricos.

Os cães domésticos apresentam desde crânios longos e estreitos, classificados como dolicocefálicos, a crânios largos e curtos, pertencentes aos braquicefálicos. Formatos intermediários compõem o grupo de cães mesaticefálicos. Os índices cefálico e crânio facial caracterizam o tipo de crânio nestes animais ${ }^{(1)}$. O cão doméstico (Canis familiaris) e o Lobo Guará (Chrysocyon brachyurus) compõem a família Canidae, mas não há classificação quanto ao tipo de crânio para a espécie silvestre.

O lobo-guará destaca-se como o maior representante silvestre da família Canidae na América do Sul, podendo atingir até $30 \mathrm{~kg}$. Ocorre em seis países da América Latina: Argentina, Bolívia, Brasil, Paraguai, Peru e Uruguai ${ }^{(5)}$.

Os objetivos do presente trabalho foram obter mensurações em crânios de C. brachyurus adultos, caracterizar valores craniométricos para a espécie e observar se a classificação craniométrica de cães domésticos é adequada para o lobo-guará.

\section{Material e Métodos}

Foram utilizados sete crânios de lobo-guará adultos, sem distinção de sexo, procedentes do acervo osteológico do Laboratório de Ensino e Pesquisa em Animais Silvestres - LAPAS/FAMEV/UFU, que possui acordo de cooperação técnica com o IBAMA de número 002 2011. Os crânios estudados foram preparados por meio da técnica de maceração com posterior clarificação em água oxigenada.

Os critérios, medidas e índices cefálicos utilizados neste estudo foram baseados em trabalhos de avaliação craniométrica em cães domésticos ${ }^{(2,6-9)}$. As avaliações foram realizadas com auxílio de paquímetro manual Starrett $125 \mathrm{MEB}$. Todas as medidas foram realizadas pelo mesmo observador, com três repetições em momentos distintos em cada crânio, a fim de evitar qualquer manipulação dos resultados. Os dados foram dispostos em tabelas para cálculo de média e desvio-padrão.

Os pontos craniométricos determinados para a realização das medidas foram os seguintes:

- Básio (B): relativo ao ponto médio da margem ventral do forame magno;

- Bregma (BR): compreende a junção no plano mediano das suturas fronto-parietais direita e 
esquerda;

- Eurio (E): ponto mais lateral do neurocrânio;

- Ínio (I): relativo ao ponto central na protuberância occipital externa;

- Násio $(\mathrm{N})$ : referente à junção, no plano mediano, das suturas fronto-nasais direita e esquerda;

- Pogônio (P): parte mais rostral da mandíbula, na sínfise, no ponto mais próximo entre as raízes dos dentes incisivos inferiores centrais;

- Próstio (PR): ponto na extremidade rostral da sutura interincisiva, entre as raízes dos dentes incisivos superiores centrais;

- Rínio (R): ponto mais rostral na sutura dos ossos nasais;

- Zígio (Z ): ponto mais lateral do arco zigomático.

A partir dos nove pontos craniométricos relatados, doze medidas lineares do crânio (de 'a' a ' $m$ ') e seis da área occipital (de 'n' a 's') foram obtidas:

- Altura do crânio (a): ponto médio do meato acústico externo ao bregma;

- Altura do triângulo occipital (q): altura máxima do triângulo occipital;

- Altura do forame magno (r): altura máxima do forame magno;

- Comprimento do crânio (b): ínio ao próstio;

- Comprimento do neurocrânio (c): ínio ao násio;

- Comprimento do viscerocrânio (d): násio ao próstio;

- Comprimento da base do crânio (g): básio ao próstio;

- Comprimento condilobasal (h): próstio às bordas caudais dos côndilos occipitais;

- Comprimento do focinho (i): ponto médio da borda oral da órbita ao próstio;

- Comprimento dos ossos nasais (j): násio ao rínio;

- Comprimento da mandíbula (1): margem caudal do côndilo ao pogônio;

- Comprimento do palato (m): espinha nasal caudal do palatino ao próstio;

- Comprimento da incisura dorsal ao forame magno (s): ponto médio da incisura dorsal ao forame magno;

- Largura do neurocrânio (e): distância entre eurios esquerdo e direito;

- Largura zigomática (f): largura entre zigios esquerdo e direito;

- Largura entre processos jugulares (n): largura máxima encontrada entre os processos jugulares;

- Largura entre os côndilos occipitais (o): largura máxima entre os côndilos occipitais;

- Largura do forame magno (p), largura máxima do forame magno.

Cinco índices cefálicos foram calculados utilizando-se as medidas lineares pré-estabelecidas, conforme demonstrado na Tabela 1 . 
Tabela 1. Índices cefálicos e respectivas fórmulas utilizadas para cálculos craniométricos em Canideos

\begin{tabular}{ll}
\hline \multicolumn{1}{c}{ Índices } & \multicolumn{1}{c}{ Fórmulas } \\
\hline Cefálico & largura zigomática $\times 100 /$ comprimento do crânio \\
Crânio facial & comprimento do crânio/ comprimento do viscerocrânio \\
do Neurocrânio & largura do neurocrânio $\times 100 /$ comprimento do neurocrânio \\
Facial & largura zigomática $\times 100 /$ comprimento do viscerocrânio \\
Basal & largura do neurocrânio $\times 100 /$ comprimento basal \\
do Forame magno & altura do forame magno $\times 100 /$ maior largura do forame magno \\
\hline
\end{tabular}

\section{Resultados}

Os pontos craniométricos e medidas cefálicas em lobo-guará são demonstradas nas Figuras 1 a 5.

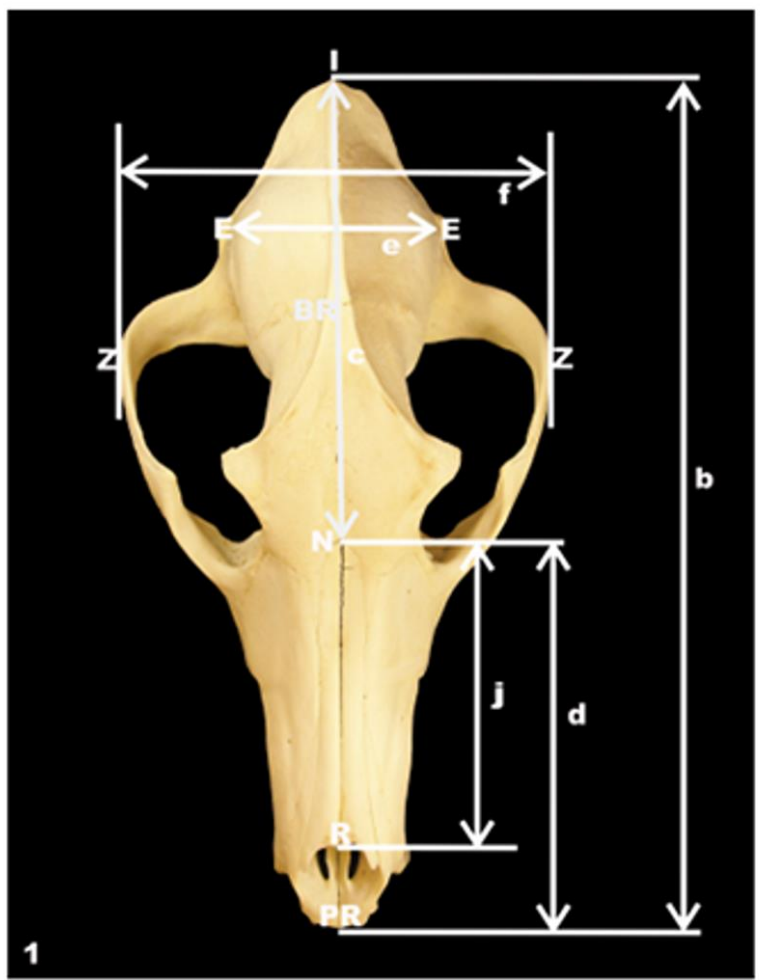

Figura 1. Fotomacrografia de crânio de lobo-guará Chrysocyon brachyurus Illiger, 1815, vista dorsal. BR (bregma), $\mathrm{E}$ (eurio), I (ínio), $\mathrm{N}$ (násio), PR (próstio), $\mathrm{R}$ (rínio), $\mathrm{Z}$ (zígio), $\mathrm{b}$ (comprimento do crânio), c (comprimento do neurocrânio), d (comprimento do viscerocrânio), e (largura do neurocrânio), $\mathrm{f}$ (largura zigomática) e $\mathrm{j}$ (comprimento dos ossos nasais). 


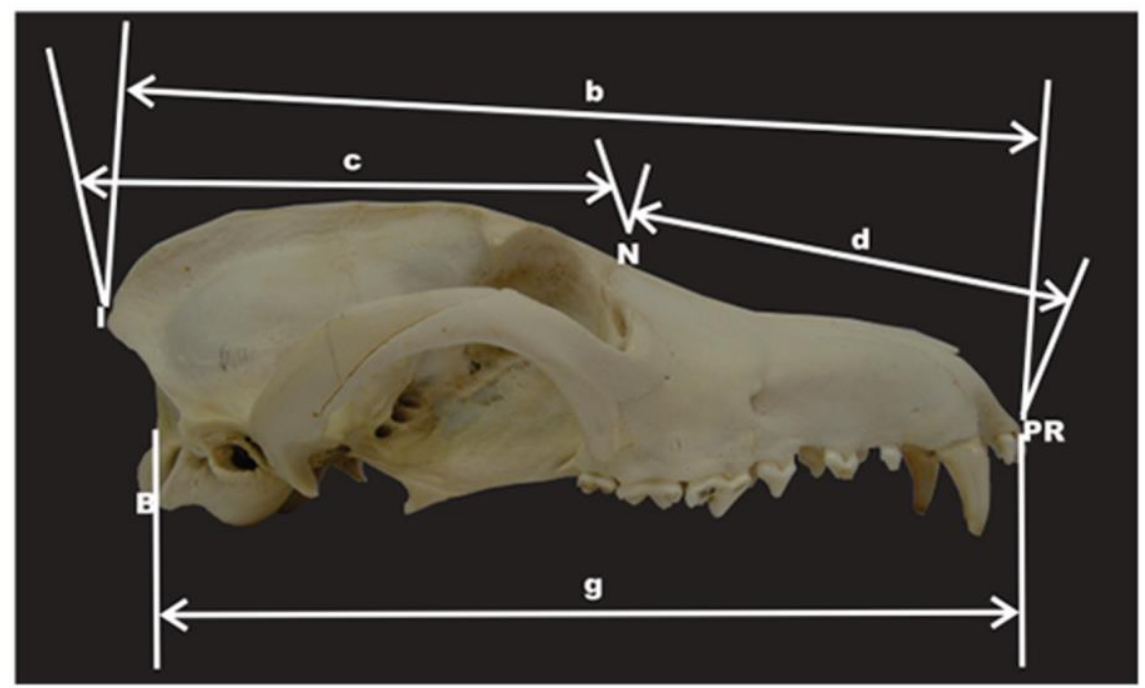

Figura 2. Fotomacrografia de crânio de lobo-guará Chrysocyonbrachyurusilliger, 1815, vista lateral. I (inio), N (násio), PR (próstio), b (comprimento do crânio), c (comprimento do neurocrânio), $\mathrm{d}$ (comprimento do viscerocrânio) e g (comprimento da base do crânio).

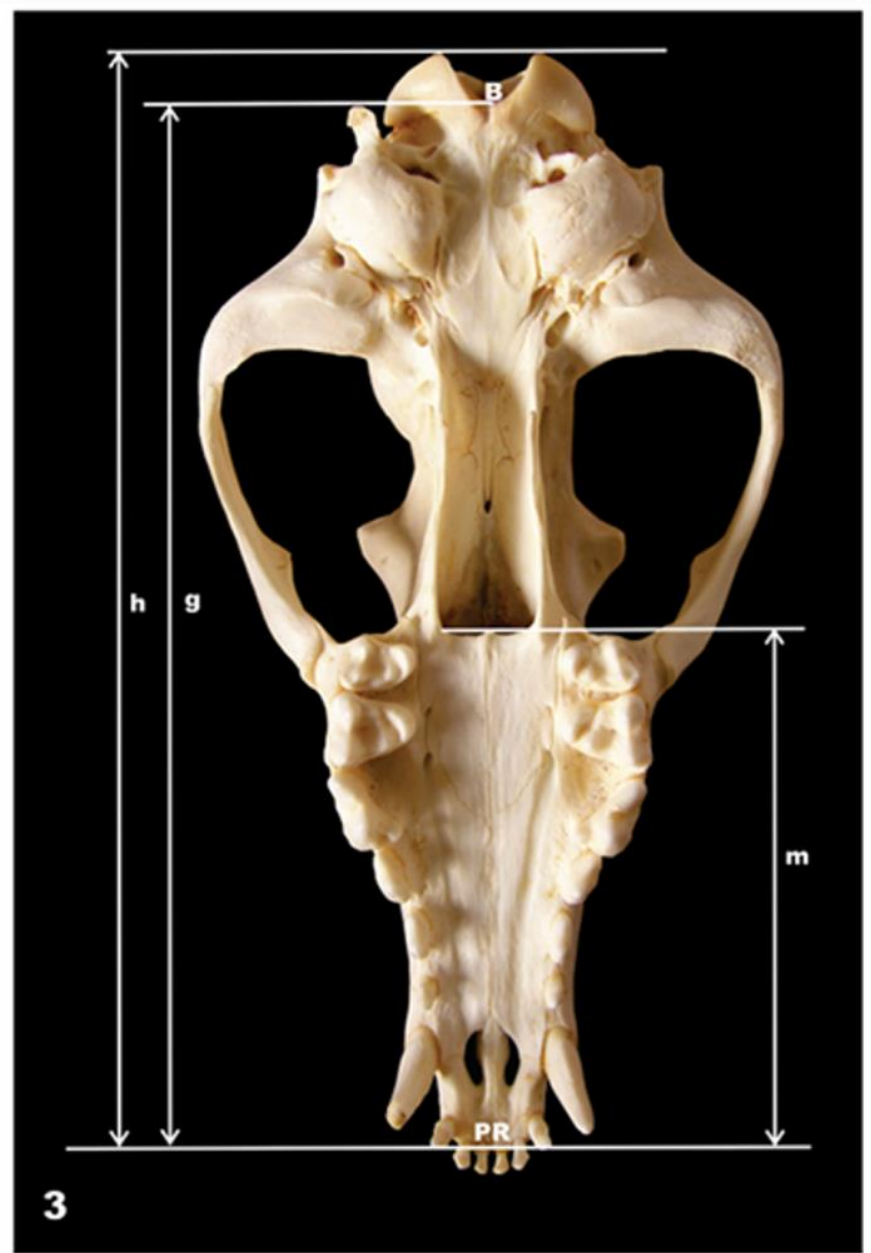

Figura 3. Fotomacrografia de crânio de lobo-guará Chrysocyon brachyurus Illiger, 1815, vista ventral.B (básio), $\mathrm{PR}$ (próstio), g(comprimento da base do crânio), h (comprimento condilobasal) e m (comprimento do palato). 


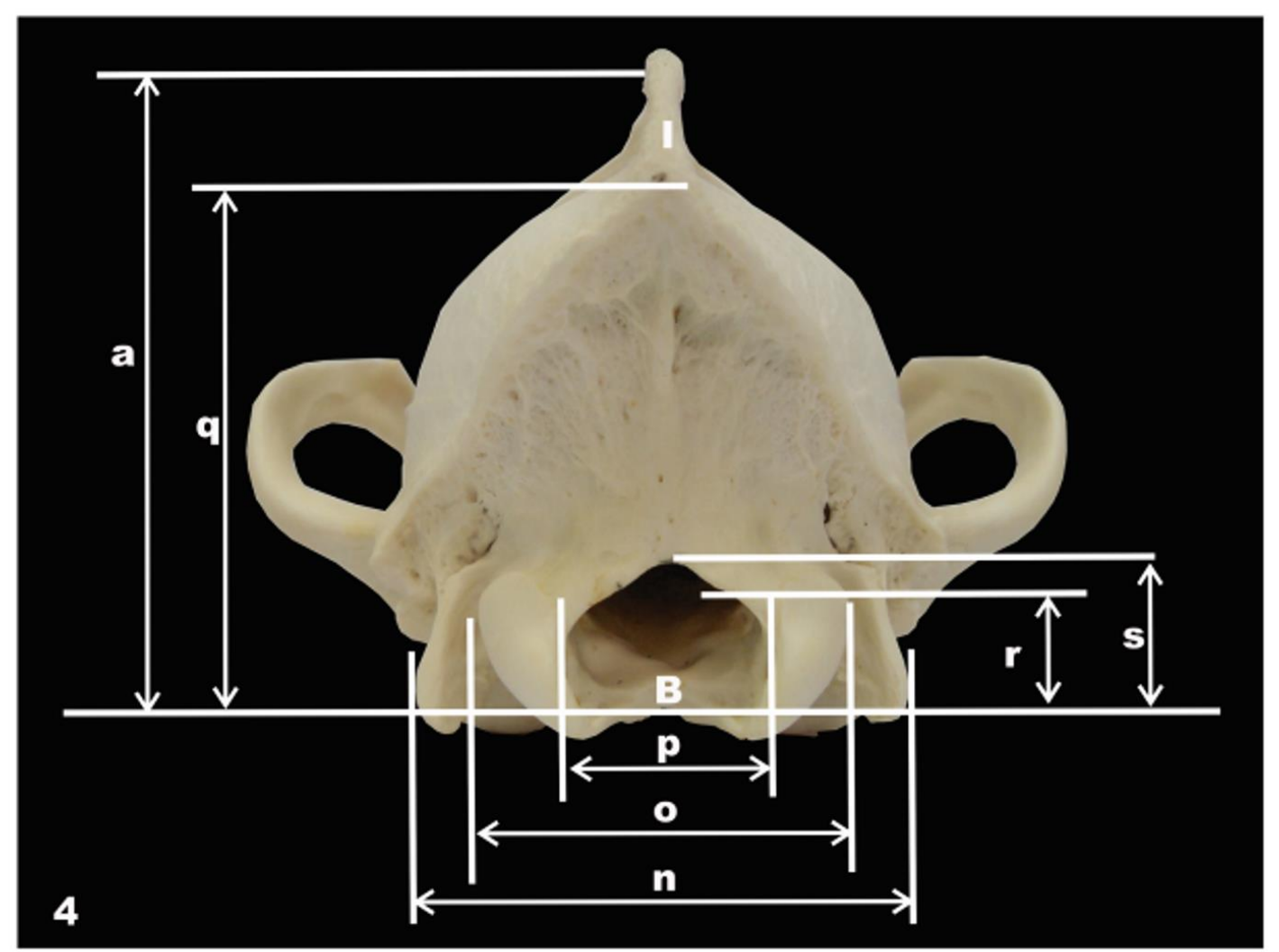

Figura 4. Fotomacrografia de crânio de lobo-guará Chrysocyon brachyurus Illiger, 1815, vista caudal. B (básio), I (ínio), a (altura do crânio), n (largura entre processos jugulares), o (largura entre côndilos occipitais), p (largura do forame magno), q (altura do triângulo occipital), r (altura do forame magno) es (comprimento da incisura dorsal ao forame magno).

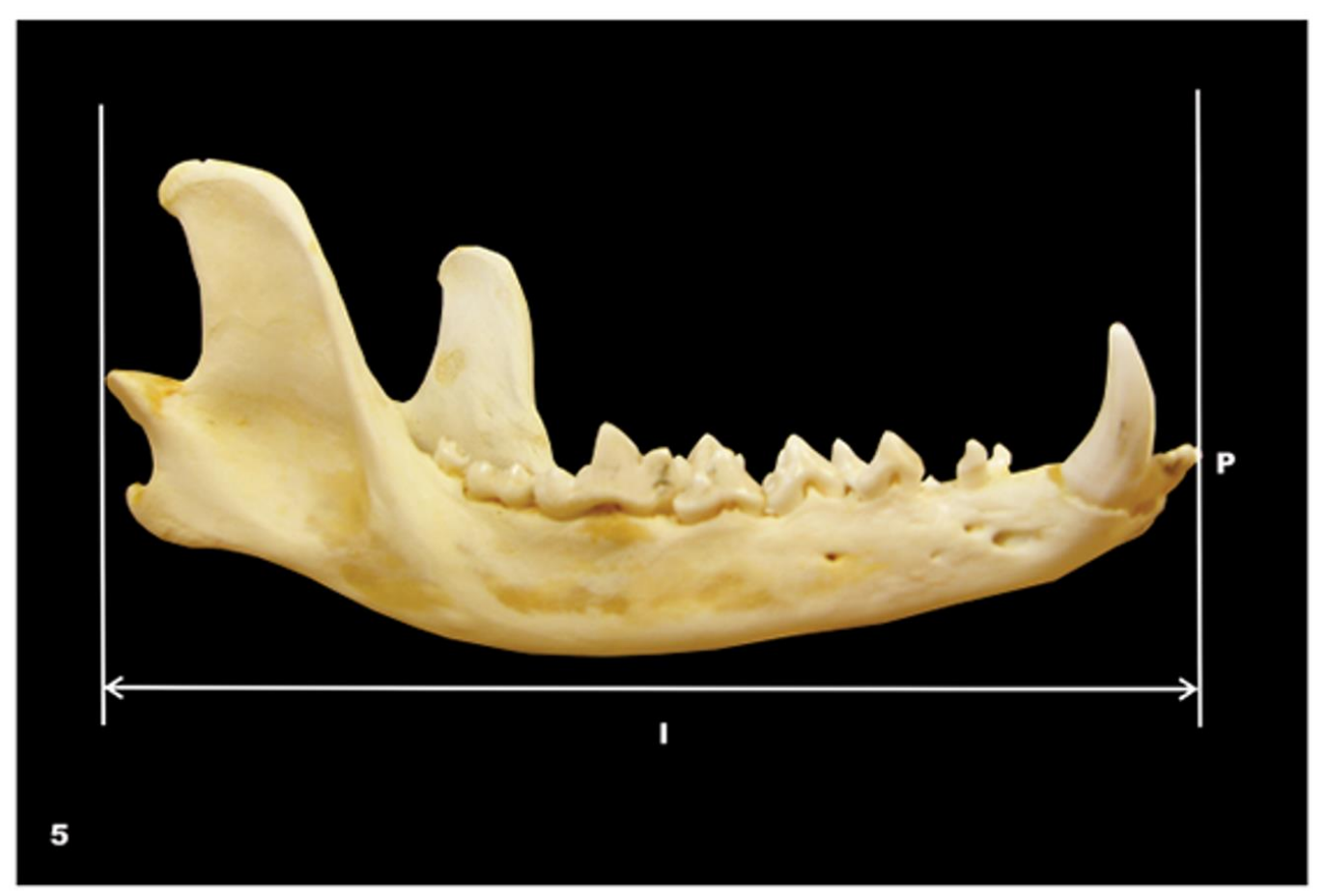

Figura 5. Fotomacrografia de mandibula de lobo-guará Chrysocyon brachyurus Illiger, 1815, vista lateral. P (pogônio) e 1 (comprimento da mandíbula). 
Os valores encontrados para as medidas craniométricas estão demonstrados na Tabela 2 como média \pm desvio-padrão. O comprimento médio do crânio foi de $126,6 \mathrm{~mm} \pm 5,29$ e a largura zigomática de $121,87 \mathrm{~mm} \pm 6,30$.

Tabela 2 - Medidas craniométricas em crânios de lobo-guará Chrysocyon brachyurus Illiger, 1815 , em milimetros

\begin{tabular}{llc}
\hline Medida & Média & Desvio-padrão \\
\hline Comprimento do Crânio & 126,76 & 5,29 \\
Comprimento do Neurocrânio & 104,45 & 2,87 \\
Largura do Neurocrânio & 64,41 & 2,01 \\
Comprimento do Viscerocrânio & 121,80 & 4,71 \\
Altura do Crânio & 73,30 & 3,10 \\
Largura Zigomática & 121,87 & 6,30 \\
Comprimento Base do Crânio & 216,41 & 7,77 \\
Comprimento Condilobasal & 226,22 & 7,03 \\
Comprimento do Focinho & 113,13 & 4,02 \\
Comprimento dos Ossos Nasais & 86,13 & 2,97 \\
Comprimento da Mandibula & 177,61 & 5,44 \\
Comprimento do Palato & 116,01 & 3,17 \\
\hline
\end{tabular}

Os resultados das mensurações realizadas na área occipital são mostrados na Tabela 3.

Tabela 3. Medidas craniométricas da área occipital em lobo-guará Chrysocyon brachyurus Illiger, 1815 , em milimetros

\begin{tabular}{llc}
\hline Medidas & Média & Desvio-padrão \\
\hline Largura entre processos jugulares & 54,83 & 2,28 \\
Largura entre côndilos occipitais & 41,85 & 1,63 \\
Largura do forame magno & 22,54 & 1,74 \\
Altura do triângulo occipital & 57,97 & 1,45 \\
Altura do forame magno & 20,02 & 0,95 \\
Comprimento da incisura dorsal do forame magno & 21,12 & 1,14 \\
\hline
\end{tabular}

Os valores encontrados pelo cálculo dos índices craniométricos estão na Tabela 4.

Tabela 4. Índices cefálicos obtidos em lobo-guará Chrysocyon brachyurus Illiger, 1815, em milimetros.

\begin{tabular}{lcc}
\hline Índices & Média & Desvio-padrão \\
\hline Índice cefálico & 96,17 & 3,89 \\
Índice crânio facial & 1,06 & 0,20 \\
Índice neurocrânio & 61,68 & 1,83 \\
Índice facial & 100,06 & 3,36 \\
Índice basal & 29,79 & 1,18 \\
Índice do forame magno & 89,25 & 7,82 \\
\hline
\end{tabular}




\section{Discussão}

$\mathrm{Na}$ literatura, há poucos trabalhos que apresentam resultados equivalentes às 18 medidas lineares obtidas neste estudo. O comprimento do crânio do lobo-guará equivale a 126,76 $\pm 5,29 \mathrm{~mm}$. Shimming e Pinto e Silva ${ }^{(2)}$ encontraram para espécimes de cães mesaticefálicos sem raça definida (SRD) $172,72 \pm 16,22 \mathrm{~mm}$. Para filhotes dolicocefálicos da raça Pastor Alemão, Onar ${ }^{(10)}$ encontrou média de comprimento do crânio equivalente a 113,96 mm para animais entre 45 e 65 dias de idade e 146,38 mm para cães entre 65 e 105 dias de idade.

A largura zigomática observada no lobo-guará foi $121,87 \pm 6,30 \mathrm{~mm} ; 95,44 \pm$ 7,85 mm e 65,52 mm e 73,54 mm para cães dolicocefálicos ${ }^{(10)}$. Os resultados das mensurações dos comprimentos da mandíbula, da base do crânio, do viscerocrânio, condilobasal, do focinho e do palato apresentaram as seguintes variações 50,$92 ; 76,17 ; 42,56 ; 76,9 ; 43,29$ e 39,17, em milímetros, respectivamente, quando comparados aos resultados observados por Shimming e Pinto e Silva ${ }^{(2)}$.

Segundo Getty ${ }^{(1)}$, o índice cefálico de cães braquicefálicos pode chegar a 90, sendo que para raças mesaticefálicas apresenta variações, 70 para o fox-terrier e 70 a 75 para o pomeriano branco. No presente estudo, o resultado encontrado para este índice no lobo-guará foi 96,147 $\pm 3,89$, superior à classificação de braquicefálicos de Getty ${ }^{(1)}$. No entanto, ele também apresenta o índice crânio facial como fundamental para a classificação do tipo de crânio. Em cães braquicefálicos, este índice pode ser superior a 3, o que não condiz com o resultado 1,06 \pm 0,20 observado para lobo-guará, próximo a 1,42 observado em dolicocefálicos ${ }^{(10)}$.

Desse modo, verificou-se que a classificação do tipo de crânio não se adequa ao lobo-guará, pois o animal receberia a classificação de braquicefálico, segundo o índice cefálico, e de dolicocefálico, segundo o índice crânio-facial. Essa diferença nos valores encontrados entre cão doméstico e loboguará pode estar relacionada ao menor comprimento do crânio associado à maior largura zigomática, características observadas na espécie silvestre. De modo ainda mais divergente, de acordo com Kunzel et al. ${ }^{(11)}$, observa-se a classificação de crânios em gatos domésticos determinada também pelo fenótipo característico dos formatos triangular, cuneiforme e arredondado. Segundo Onar ${ }^{(10)}$, pode haver variações nos índices craniométricos de acordo com a idade do animal.

Alpak et al. ${ }^{(12)}$ obtiveram, para a espécie braquicefálica Pekingese, índice cefálico equivalente a 97,16 e 2,20 para o índice crânio facial. Os valores próximos ao lobo-guará para o índice crânio facial no referido trabalho foram equivalentes às raças Kangal Dog, Doberman, German Shepherd e Cocker Spaniel, todos dolicocefálicos. Os autores encontraram variações intraespecíficas para a raça Mixed Breed, sendo dois crânios classificados como dolicocefálicos e um terceiro como mesaticefálico.

Para o índice do neurocrânio, cães das raças Kangal Dog, Boxer e Clumber Spaniel apresentaram, no estudo de Alpak et al. ${ }^{(12)}$, valores muito próximos ao observado no lobo-guará. Ainda, para o índice facial o crânio da raça Mixed Breed, classificado como mesaticefálico, foi o que mais se aproximou do lobo-guará, sendo 100,44 encontrado para o primeiro e 100,06 para a espécie silvestre. 


\section{Conclusões}

Os pontos craniométricos determinados para o lobo-guará foram similares aos descritos na literatura para o cão doméstico, sendo as medidas calculadas de forma equivalente. A classificação em braquicefálico, dolicocefálico ou mesaticefálico não se adequa ao crânio de $C$. brachyurus.

\section{Referências}

1 Getty R. Anatomia dos animais domésticos. 5. ed. Rio de Janeiro: Guanabara Koogan, 1986;2:1377-1391.

2 Shimming BC, Pinto e Silva JRC. Craniometria em cães (Canis familiaris). Aspectos em crânios mesaticéfalos. Braz. J. Vet. Res. Anim. Sci., São Paulo. 2013;50(1):5-11.

3 Kalita A, Sarma K, Shalini MMS \& Ahmad B. Craniometrical study in Bakarwali goat. Indian J. Anim. Hlth. 2004;43(2):180-184.

4 Christiansen P, Adolfssen JS. Bite forces, canine strength and skull allometry in carnivores (Mammalia, Carnivora). J. Zool. 2005;266:133-151.

5 Paula RC, Medici P, Morato RG. (ORG.). Plano de Ação para a Conservação do Lobo-Guará - Análise de Viabilidade Populacional e de Habitat. - Brasília: Centro Nacional de Pesquisas para Conservação dos Predadores Naturais - CENAP/ICMBio. 2008-2009:158p.

6 Simoens P, Poels P, Lauwers, H. Morphometric analysis of the foramen magnum in Pekingese dogs. American Journal of Veterinary Research. 1994;55(1):34-39.

7 Onar V, Mutu R, Kahvecioglu K. O. Morphometric analysis of the foramen magnum in German Shepherd dogs (Alsatians). Annals of Anatomy. 1997;179(6):563-568.

8 Onar V, Günes H. On the variability of skull shape in German Shepherd (Alsatian) puppies. Anatomical Record. 2003;272A(1):460-466.

9 Janeczek M, Chrószcz A, Onar V, Pazvant G, Pospieszny N. Morphological analysis of the foramen magnum of dogs from the iron age. Anatomia, Histologia, Embryologia. 2008;37(5):359-361.

10 Onar, V. A Morphometric Study on the Skull of the German Shepherd Dog. Anatomia, Histologia, Embryologia. 1999:28(4):253 - 256.

11 Künzel W, Breit S, Oppel, M. Morphometric investigations of breed-specific features in feline skulls and considerations on their functional implications. Anatomy Histology and Embryology. 2003;32(4):218-23.

12 Alpak H, Mutuy R, Onar, V. Correlation analysis of the skull and long bone measurements of the dog. Annal sof Anatomy. 2004;186:323-330. 